\title{
EL DERECHO PENAL COMO MEDIO DE PREVENCIÓN DE LA VIOLENCIA OBSTÉTRICA EN MÉXICO. RESULTADOS AL 2018
}

\author{
Guillermo Manuel Corral-Manzano1 \\ Correo electrónico: guillermocorral@live.com.mx
}

1. Doctorando en Ciudadanía y Derechos Humanos, Universidad de Barcelona. Maestro en Política Criminal por la Universidad Nacional Autónoma de México. Licenciado en Derecho por la Universidad Tecnológica de México.

Recibido: 02/12/2018 Aceptado: 25/06/2019

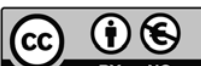

${ }^{1}$ Este trabajo se ha presentado dentro del III Congreso Internacional «Género, ética y cuidado», celebrado del 16 al 18 de mayo en Barcelona y forma parte del proyecto «Diversidad de género, masculinidad y cultura en España, Argentina y México» (FEM2015-69863-P MINECO-FEDER) del Ministerio de Economía y Competitividad de España. 


\section{RESUMEN}

\section{Introducción}

México, posteriormente a la celebración de la Convención Interamericana para Prevenir, Sancionar y Erradicar la violencia contra la Mujer, «Convención Belém do Pará» de 1994, ha pretendido adecuar su legislación interna para dar cumplimiento a las obligaciones internacionales adquiridas. El Estado de Veracruz (México), a partir del año 2010, consideró la violencia obstétrica como delito en su código penal. Tras ocho años de su tipificación, se considera oportuno revisar los efectos de su implementación.

\section{Metodología}

A través del análisis de la norma vigente, de algunos programas de salud que con motivo de la violencia obstétrica se han sugerido, y de la información sobre conciliaciones médicas, recomendaciones y denuncias que se han realizado, el presente texto pretende hacer una crítica a las medidas tomadas en México sobre violencia obstétrica, haciendo hincapié en que el derecho penal no es por si solo suficiente para prevenir esta clase de violencia.

\section{Resultados y Discusión:}

Los mecanismos adoptados por el gobierno mexicano para prevenir y erradicar la violencia obstétrica parecen quedarse a nivel normativo: no existen juicios penales por el delito de violencia obstétrica en el Estado de Veracruz a ocho años de su tipificación; las recomendaciones de los organismos de derechos humanos nacionales, que versan sobre violencia obstétrica no sugieren el uso del derecho penal para la sanción de las personas que la han ejercido y solo se contempla violencia obstétrica cuando hay muerte del feto o secuelas en el mismo; el organismo nacional de arbitraje médico, tiene en relación a la práctica médico ginecológica porcentualmente un índice de laudos condenatorios inferior a los absolutorios y/o a los convenios.

\section{Conclusiones}

La experiencia de México puede ser tomada en consideración por otros países a efecto de no incurrir en los mismos errores o adoptar medidas que generen aciertos. Se requiere poner en el foco de atención la normalización que existe respecto a este tipo de violencia, así como la falta de medidas tendentes a una sanción de las prácticas de violencia obstétrica institucional, que permea en el poco interés que se demuestra en el respeto o aplicación de protocolos específicos diseñados para prevenirla.

Palabras clave: México; violencia obstétrica; derecho; arbitraje médico. 


\section{ABSTRACT}

\section{Introduction}

Mexico, following the conclusion of the Inter-American Convention on the Prevention, Punishment and Eradication of Violence against Women, «Convention Belém do Pará» of 1994, has sought to adapt its domestic legislation to comply with the corresponding international obligations. The state of Veracruz (Mexico) has considered obstetric violence as a crime in its criminal code since 2010. After eight years, it is time to review the effects of its implementation.

\section{Methodology}

Through the analysis of the current norm, some health programs that have been suggested as a result of the obstetric violence, and information on medical conciliations, recommendations and denunciations, this text intends to criticize the measures taken by Mexico on obstetric violence, emphasizing that criminal law alone is not enough to prevent this kind of violence.

\section{Results and Discussion}

The mechanisms adopted by the Mexican government to prevent and eradicate obstetric violence seem to remain at the normative level: there are no criminal trials for the crime of obstetric violence in the state of Veracruz, eight years after its classification; the recommendations of national human rights organizations, which deal with obstetric violence, do not suggest the use of criminal law for the punishment of those who have exercised it, and obstetric violence is contemplated only when the fetus dies or is seriously affected by it; the national body of medical arbitration has, in relation to gynecological medical practice, a percentage of lower rate of convictions as compared with acquittals and/or agreements.

\section{Conclusions}

The experience of Mexico can be taken into consideration by other countries in order not to repeat the same mistakes and adopt measures that generate more positive outcomes. It is necessary to focus on the process of «normalization» with regard to this type of violence-as well as on the lack of measures that punish the practices of institutional obstetric violence-which tells us about the little interest shown in the respect or application of specific protocols designed to prevent it.

Keywords: Mexico; Obstetric Violence; Law; Medical Arbitration. 


\section{INTRODUCCIÓN}

Uno de los problemas al hablar de violencia obstétrica y de género es que existe un discurso hegemónico de la violencia. Al referirnos a «violencia», lo primero que se piensa son los actos bélicos como la guerra, la tortura o el daño físico, entre otros. La normalización que existe de otros tipos de violencia como la simbólica, o la que se produce a través de prácticas médicas que se adueñan o patologizan los procesos reproductivos de las mujeres, aunado al discurso hegemónico de la violencia, producen que otros discursos queden marginados. Esta normalización genera que, pese a contar con un marco normativo que pretenda ser garante de que las mujeres vivan una vida libre de violencia obstétrica, este no logre su cometido, en virtud de que queda en desuso o sencillamente es inobservado. Esto puede suceder porque las víctimas de esta clase de violencia desconozcan del marco normativo o sencillamente porque, pese a considerar que el trato recibido durante su embarazo, parto o puerperio no fue el más adecuado, deciden no interponer ningún tipo de queja, querella o denuncia, en virtud de que desean dejar ese episodio en el olvido. También el seguimiento que se da por parte del gobierno a la implementación de los protocolos para un parto humanizado, o de que los nosocomios cuenten con el personal sensibilizado y suficiente, equipo e instalaciones adecuados, genera que la inobservancia a dichos protocolos vaya de la mano de la invisibilización y normalización de la violencia obstétrica.

\section{De la violencia obstétrica y la obligación internacional de prevenirla}

A partir de la «Convención de Belém do Pará» de 1994, los Estados miembros de la Organización de los Estados Americanos (OEA), se comprometieron a tomar las medidas necesarias para prevenir, sancionar y erradicar la violencia contra la mujer. En particular, por lo que respecta a la violencia obstétrica, el Mecanismo de Seguimiento de la Convención de Belém do Pará en el año 2012, en su Segundo Informe Hemisférico sobre la Implementación de la Convención, insta a los Estados miembro a

adoptar disposiciones que penalicen la violencia obstétrica. Establecer por los medios apropiados los elementos de lo que constituye un proceso natural antes, durante y después del parto, sin excesos ni arbitrariedad en la medicación, que garantice la manifestación del consentimiento libre y voluntario de las mujeres en los procedimientos vinculados a su salud sexual 
y reproductiva. Adoptar una perspectiva intercultural que respete las costumbres y pautas culturales de las mujeres indígenas y afrodescendientes en los centros de salud. (MESECVI, 2012, p. 98)

La violencia obstétrica, en palabras de Júlia Martin y Monserrat Payà, podemos entenderla como «el conjunto de actuaciones asistenciales y prácticas técnico-sanitarias que se desarrollan a lo largo del proceso de atención sanitaria que se traducen en una apropiación del cuerpo u de los procesos reproductivos de las mujeres por parte de los prestadores de salud» (Martin y Payá, 2018). Respecto a dicho concepto, se ha indicado que la violencia obstétrica se manifiesta esencialmente en seis focos de interés: negligencia, violencia física, violencia sexual, violencia institucional y medicina defensiva (Cruz, 2015, p. 2).

La Comisión Nacional de Derechos Humanos de México (CNDH), en su recomendación 06/2017, en su párrafo 53, señala que la violencia obstétrica es

una modalidad de la violencia institucional y de sexo, cometida por algunos prestadores de servicios de la salud, por una deshumanizada atención médica a las mujeres durante el embarazo, parto o puerperio que le genere una afectación física, psicológica o moral, que incluso llegue a provocar la pérdida de la vida de la mujer o, en su caso, del producto de la gestación o del recién nacido, derivado de la prestación de servicios médicos, abuso de medicalización y patologización de procedimientos naturales, entre otros. (Recomendación de la CNDH, 06/2017, del 28 de febrero de 2017)

La citada Comisión indica que «observa con preocupación que en ocasiones la violencia obstétrica ha sido naturalizada por personal médico, y la sociedad en su conjunto» (Recomendación de la CNDH, 31/2017, del 31 de julio de 2017).

\section{Del delito de violencia obstétrica y de otras legislaciones que la contemplan en México.}

En ocasiones anteriores, ya he manifestado una postura particular sobre el hecho de que la penalización de la violencia obstétrica como única medida para su prevención no es el 
camino idóneo. ${ }^{2}$ En el presente artículo, la intención es analizar de manera más detallada el sistema normativo y de políticas públicas desarrollado en México con respecto de la violencia obstétrica, a efecto de que pueda servir como referente de experiencia a considerar al tomar medidas respecto a la prevención de este tipo de violencia.

México, en virtud de la «Convención Belém do Pará», ha pretendido adecuar su legislación interna para dar cumplimiento a las obligaciones contraídas. Sin embargo, en lo que respecta a la violencia obstétrica, a la fecha no todos los códigos penales de las diversas entidades federativas contemplan el delito en particular. ${ }^{3}$ Es precisamente en el año 2010 cuando el Código Penal del Estado de Veracruz tipifica por primera vez la violencia obstétrica en México, conceptualizándola en el artículo 369 fracción III como el «acto u omisión que afecta la autonomía y la capacidad de decidir de las mujeres sobre su sexualidad y procesos reproductivos» (Veracruz), sancionando en su artículo 363 a quien

I. No atienda o no brinde atención oportuna y eficaz a las mujeres en el embarazo, parto, puerperio o. en emergencias obstétricas; II. Altere el proceso natural del parto de bajo riesgo, mediante el uso de técnicas de aceleración, sin obtener el consentimiento voluntario, expreso e informado de la mujer; III. No obstante existir condiciones para el parto natural, practique el parto por vía de cesárea, sin obtener el consentimiento voluntario, expreso e informado de la mujer; IV. Acose o presione psicológica u ofensivamente a una parturienta, con el fin de inhibir la libre decisión de su maternidad; V. Sin causa médica justificada, obstaculice el apego del niño o la niña con su madre, mediante la negación a ésta de la posibilidad de cargarle o de amamantarle inmediatamente después de nacer; y VI. Aun cuando existan los medios necesarios para la realización del parto vertical, obligue a la mujer a parir

\footnotetext{
${ }^{2}$ Para más información, véase: Corral, Guillermo. ¿Es necesario tipificar la violencia obstétrica? En: Goberna-Tricas, Josefina; Boladeras, Margarita (Coord.). El concepto «Violencia Obstétrica» y el debate sobre la atención al nacimiento. Barcelona: Tecnos, 2018, pp. 93-101.

${ }^{3}$ A efecto del presente artículo se revisaron los 33 códigos penales vigentes en México al mes de octubre del año 2018. Es menester el indicar que la Comisión Nacional de Derechos Humanos de México, en fecha 31 de julio del año 2017 en su recomendación 31/2017, realiza un mapeo similar respecto de las leyes y códigos vigentes en México que versan sobre violencia obstétrica a julio del 2017. La citada comisión revisa el marco normativo publicado hasta dicha fecha, siendo su revisión concordante con la información presentada durante el Workshop «El concepto violencia obstétrica» en Barcelona, el cinco de mayo del 2017 y reseñada en la nota al pie anterior.
} 
acostada sobre su columna y con las piernas levantadas o en forma distinta a la que sea propia de sus usos, costumbres y tradiciones obstétricas.

En la actualidad, solo los códigos penales de los Estados de Chiapas, Estado de México, Quintana Roo, Guerreo y el ya citado código penal de Veracruz, son los que contemplan como delito a la violencia obstétrica. ${ }^{4}$ Por su parte, para el año 2011, el Código Penal para el Distrito Federal agregó al artículo 201 la fracción VI, en el cual se sanciona la violencia contra los derechos reproductivos dentro de la violencia familiar, el cual consiste en «toda acción u omisión que limite o vulnere el derecho de las mujeres a decidir libre y voluntariamente sobre su función reproductiva, en relación con el número y esparcimiento de los hijos, así como el acceso a servicios de aborto seguro en el marco previsto en los ordenamientos relativos para la interrupción legal del embarazo, a servicios de atención prenatal, así como a servicios obstétricos de emergencia» (Distrito Federal), lo cual, si bien no contemplaría todos los casos en los que se puede manifestar la violencia obstétrica, es un comienzo. Los códigos penales de los Estados de Oaxaca y Coahuila contemplan la misma figura en los mismos términos que en el código penal para el Distrito Federal, ahora Ciudad de México.

El Estado mexicano ha pretendido hacer frente a la violencia obstétrica desde diferentes frentes. La propia CNDH en México, en su recomendación 31/2017 ${ }^{5}$ ha pretendido destacar los avances que se han tenido al respecto. Dentro de ellos, cabe resaltar que, para el mes de abril del año 2014, la Secretaria de Gobernación en México a través del denominado «Programa Integral para Prevenir, Atender, Sancionar y Erradicar la Violencia contra las Mujeres 2014-2018», en sus estrategias 1.1 y 2.3, establece que se debe

Promover la armonización de la legislación nacional con tratados y convenciones internacionales, eliminando disposiciones discriminatorias en contra de las mujeres [...] fortalecer los servicios para la detección oportuna de la violencia contra las mujeres en el Sistema Nacional de Salud a través de

\footnotetext{
${ }^{4}$ El Estado de Chiapas lo contempló a partir del año 2014, el Estado de México agregó el delito de violencia obstétrica a finales del año 2016, mientras que los Estados de Quintana Roo y Guerrero agregaron el tipo penal hacia finales del año 2017.

${ }^{5}$ Se recomienda ampliamente dar lectura a la recomendación 31/2017, en la cual se pueden observar la situación actual de la violencia obstétrica en México a los ojos de la Comisión Nacional de Derechos Humanos.
} 
la promoción de políticas institucionales para erradicar la violencia de género que ocurre en los servicios de salud, incluyendo violencia obstétrica. (p. 19)

El 19 de diciembre del año 2016 se modificó el Reglamento de la Ley General de salud con el objeto de «regular la atención médica que debe brindarse a las mujeres que presentan una urgencia obstétrica con independencia de su derechohabiencia o afiliación a cualquier esquema de aseguramiento» (Recomendación de la CNDH, 31/2017, de 31 de julio de 2017, p. 18). En el mismo sentido, se desarrolló en el año 2008 por parte de la Secretaria de Salud el denominado «Modelo de Atención a las Mujeres durante el Embarazo, Parto y Puerperio con Enfoque Humanizado Intercultural y Seguro», el cual propone que «la mujer sea la protagonista de su parto y se involucre la participación de las parteras y los parteros tradicionales» (p. 19). Dicho programa, a decir de la propia comisión de derechos humanos, solo ha sido implementado en 14 hospitales de toda la República Mexicana, en específico en los Estados de Baja California Norte, Campeche, Colima, Chihuahua, Durango, Ciudad de México, Hidalgo y el Estado de México (p. 74).

Así mismo, la CNDH, en la multicitada recomendación 31/2017, indica que, 19 de 29 médicos encuestados en Puebla «manifiestan no tener suficiente información de violencia obstétrica o de las herramientas para hacerle frente o evitarla» (p. 16), o que, de 323 encuestas a mujeres en Oaxaca, se desprende que «las prácticas de atención durante el trabajo de parto, parto y puerperio inmediato, se realizan en muchos casos sin apego a los estándares nacionales y/o a las recomendaciones de la OMS» (p. 17).

\section{MATERIAL Y MÉTODO}

A efecto del presente artículo, se hizo revisión de las recomendaciones que la CNDH de México ha realizado respecto a casos de violencia obstétrica ${ }^{6}$ : Se revisaron los 33 códigos penales vigentes en México al mes de octubre del año 2018. Se revisó cada código penal y leyes para el acceso a una vida libre de violencia de cada uno de los Estados de México, así como la legislación federal, buscando en sus artículos cualquier conducta que pudiere encuadrarse dentro de lo que se entiende como violencia obstétrica, independientemente de que existiera un tipo penal especifico con dicho nombre. Así mismo, se realizaron solicitudes de información puntual mediante la Plataforma Nacional de Transparencia de

\footnotetext{
${ }^{6}$ Ver notas al pie n. 2 y 3
} 
México, a la Comisión Nacional de Arbitraje Médico en México (CONAMED) respecto a arbitrajes realizados con relación a la medicina gineco-obstetra, así como al Poder Judicial y a la Fiscalía del Estado Veracruz respecto a investigaciones y juicios en los que se investigaran hechos probablemente constitutivos del delito de violencia obstétrica. Conforme a lo anterior, a través del método inductivo y contrastando la información mencionada, se pretende señalar el avance en la prevención de la violencia obstétrica y hacer una crítica a las medidas tomadas en México sobre la violencia obstétrica, haciendo hincapié en que el derecho penal no es por si solo suficiente para prevenir esta clase de violencia, señalando que la violencia obstétrica es un problema social cuya solución dista mucho de encontrarse solo en la prevención general del derecho penal. Por último, cabe observar que uno de los problemas más grandes respecto a la misma es la normalización. El análisis realizado a las legislaciones referidas atendió a la investigación doctrinalhermenéutica.

\section{RESULTADOS Y DISCUSIÓN}

\section{Consideraciones sobre los resultados de la regulación y sanción de la violencia obstétrica en México.}

Si bien podemos observar que en México ha existido trabajo legislativo en torno al tema de la violencia obstétrica, y que la misma ya es contemplada en algunos Estados como delito, en otros sencillamente se ven vinculados a elaborar programas de prevención de la misma con respecto al Plan Nacional de Desarrollo. Al analizar las conductas que se sancionan al tenor del tipo penal de violencia obstétrica, podemos observar que solo se contemplan la violencia verbal y la negligencia. Es menester señalar que por lo que hace a la violencia sexual y violencia física, ambas se encuentran contempladas dentro de los códigos penales de los Estados, dentro de los delitos de lesiones y los referentes al libre desarrollo sexual, como lo son el delito de abuso sexual y violación.

La violencia institucional puede ser entendida como «aquella que provoca daños físicos y psicológicos a las personas, como resultado de condiciones inadecuadas en instituciones y en sistemas públicos» (Cruz, 2015, p. 2). Hablando de violencia de género, la violencia institucional ha sido conceptuada por la CNDH de México como «los actos u omisiones de las y los servidores públicos de cualquier orden de gobierno que discriminen o tengan como fin dilatar, obstaculizar o impedir el goce y ejercicio de los 
derechos humanos de las mujeres, así como su acceso al disfrute de políticas públicas destinadas a prevenir, atender, investigar, sancionar y erradicar los diferentes tipos de violencia» (Recomendación de la CNDH, 31/2017, del 31 de julio de 2017, p. 34). De este modo, podemos indicar que la violencia institucional que se debería contemplar respecto a la violencia obstétrica en el país no se encuentra prevista dentro del tipo penal.

El termino de medicina defensiva de la que nos hablan Kessier y McCLellan, citados por Cruz, es entendida como el «uso excesivo de exámenes que no necesariamente van en favor del paciente, sino más bien con la intención de proteger legalmente al médico tratante; la utilización de técnicas consideradas dañinas y con poca evidencia que respalde su efectividad» (p. 2). Podríamos considerarla parcialmente contemplada en el tipo penal de violencia obstétrica, cuando se señala la negligencia (sobre todo por lo que respecta a la práctica de cesáreas no necesarias). Sin embargo, lo cierto es que realizar un excesivo número de análisis clínicos, que no sean necesarios para dar un debido seguimiento al embarazo, y que solo tengan como finalidad el acrecentar la erogación que realice la mujer por dichos procedimientos, o que su función sea solo brindar seguridad al médico tratante de no ser posible objeto de una responsabilidad profesional sin generar ningún beneficio a la mujer, no son contemplados por el tipo penal de violencia obstétrica y, por ende, no pueden ser sancionados.

Además de esta tipificación parcial de la violencia obstétrica, al momento de solicitar información al poder judicial y al Ministerio Público del Estado de Veracruz, que es el primer Estado de México que contemplo la violencia obstétrica como delito, la respuesta obtenida se limita a indicar que, a la fecha de la solicitud, ${ }^{7}$ no se tiene registro de que se haya celebrado ningún juicio penal en el que el imputado fuera probable responsable de la comisión del delito de violencia obstétrica. Por su lado, el Ministerio Público de la entidad respondió que no tiene registro de haber ejercitado acción penal en función del delito de violencia obstétrica, pero agregan que como se observa en la Tabla 1, han existido en total seis denuncias por hechos constitutivos al delito, pero de las cuales no se ha ejercitado la acción penal.

Tabla 1. Número de denuncias por violencia obstétrica en el estado de Veracruz del año 2010 al año 2017

\footnotetext{
${ }^{7}$ Para la intervención dentro del III Congreso Internacional de «Genero, ética y cuidado», celebrado en Barcelona en mayo del 2018, se solicitó información al poder judicial y al Ministerio Público del Estado de Veracruz a través del sistema de la Plataforma Nacional de Transparencia de México.
} 


\begin{tabular}{|r|c|c|c|}
\hline AÑ & $\begin{array}{c}\text { NÚMERO DE } \\
\text { DENUNCIAS } \\
\text { POR } \\
\text { VIOLENCIA } \\
\text { OBSTÉTRICA }\end{array}$ & $\begin{array}{c}\text { NÚMERO DE } \\
\text { PROCESOS } \\
\text { PENALES QUE SE } \\
\text { HAN RADICADO } \\
\text { POR CASOS DE } \\
\text { VIOLENCIA } \\
\text { OBSTÉTRICA }\end{array}$ & $\begin{array}{c}\text { NÚMERO DE } \\
\text { CENTENCIAS } \\
\text { CONDENATORIAS } \\
\text { QUE SE HAN } \\
\text { DICTADO POR } \\
\text { VIOLENCIA } \\
\text { OBSTÉTRICA }\end{array}$ \\
\hline 2015 & 1 & 0 & 0 \\
\hline 2016 & 4 & 0 & 0 \\
\hline 2017 & 1 & 0 & 0 \\
\hline TOTAL & 6 & 0 & 0 \\
\hline
\end{tabular}

Fuente: Elaboración propia. ${ }^{8}$

De la tabla anterior se desprende que en el lapso de siete años de encontrarse previsto el delito de violencia obstétrica en el código penal del Estado de Veracruz, solamente existen seis denuncias respecto de dicho ilícito, siendo que cuatro de ellas fueron realizadas en el año 2016, una en el 2015 y una en el 2017. Es imprescindible destacar que no se brinda información de porqué en las seis denuncias realizadas por violencia obstétrica no se ejercitó acción penal alguna, es decir, conforme al sistema penal mexicano, posterior a la denuncia, la autoridad investigadora debería de hacerse de los elementos suficientes para posterior a ello ejercitar acción penal contra el probable responsable e imputarlo por la comisión de un ilícito, pero por los datos brindados, esto no fue así.

Si bien puede suponerse que no obtuvo los elementos suficientes para el ejercicio de la acción penal, el hecho de que la fiscalía no refiera qué aconteció con dichas denuncias, solo nos permite determinar que, sencillamente, el hecho de que se encuentre previsto en el código penal el ilícito no garantiza de ninguna forma la sanción al mismo. La información otorgada por la fiscalía nos obliga hacernos ciertas preguntas, como ¿Acaso no hay violencia obstétrica en el Estado de Veracruz? o ¿Se encuentra tan normalizada la violencia obstétrica que por las víctimas de este tipo de violencia no se percibe la necesidad de denunciarla? Al considerar que solo se han denunciado seis casos de posible violencia obstétrica en un lapso de siete años, y que no se cuenta con mayor información por parte de la fiscalía del Estado, sería aventurado dar una respuesta concreta a las preguntas planteadas. Sin embargo, si consideramos que en el año 2015 el 47\% de los

8 La presente tabla se ha realizado en función del oficio número 1649/2017 de la Fiscalía General del Estado de Veracruz, y el oficio UTAIPPJE/406/2017 del Poder Judicial de Veracruz, mediante los cuales se da respuesta a una solicitud de información presentada en agosto del año 2017. 
nacimientos en México eran por cesárea, y que esta cifra triplica el porcentaje recomendado por la Organización Mundial de la Salud, ${ }^{9}$ podemos suponer que la normalización de la violencia obstétrica es lo que conlleva a que exista un número tan reducido de denuncias, y no así el que no exista violencia obstétrica en el Estado.

No es solo la normalización lo que conlleva a que no se denuncie la violencia obstétrica. La desinformación de las autoridades en los Estados que tipifican la violencia obstétrica como delito posiblemente también es un factor del porqué existen tan pocas denuncias. Lo anterior se desprende del número de quejas respecto a temas relacionados con la especialidad gineco-obstetra que se han llevado a la Comisión Nacional de Arbitraje Médico en México (CONAMED), comisión que en los Cuadros Estadísticos de Controversias en Salud 1996-2017 (CONAMED, 2018) informa que, del año 1996 al año 2017, se recibieron un total de 36.475 quejas, de las cuales el 57,85\% fueron presentadas por mujeres. En particular 4.671 quejas tuvieron relación con la especialidad ginecoobstetra, y de estas se registra que solo 195 fueron con relación al embarazo, mientras que 2.033 fueron relacionadas por cuestiones relativas al parto o al puerperio inmediato. Por lo anterior, podemos observar que existen una cantidad considerable de casos en los cuales las mujeres piden la intervención de este organismo cuando existen inconformidades en la práctica médico-obstétrica.

En la búsqueda de respuestas a la violencia obstétrica, y en particular a la prevención de la misma, si la CONAMED fuera el camino preciso para generar sanciones, aunque fueran administrativas en el caso de que los profesionales de la salud incurrieran en este tipo de violencia, o para sancionar a las instituciones públicas o privadas de salud por no contar con las instalaciones necesarias o personal calificado para evitar este tipo de violencia, no habría ningún problema. Es decir, desde la postura particular de considerar que el derecho penal no es el camino adecuado para la prevención de la violencia obstétrica, el hecho de que otro organismo se dedicare a la prevención efectiva y sanción administrativa sería positivo, solo en caso de que dicho organismo mostrare resultados. Sin embargo, de la información solicitada a dicha comisión también se desprende que muchos de los casos atendidos quedan sin conclusión como se puede observar en la Tabla 2.

9 Para más información véase: Cruz, Ángeles (2015), «Por cesárea, 47\% de nacimientos, el triple de lo recomendado por la OMS», La Jornada, 26 de octubre, México. 
Tabla 2. Modalidad de conclusión de las quejas relacionadas a la especialidad ginecoobstetra por parte de la CONAMED del año 1996 al 2017.

\begin{tabular}{|l|l|}
\hline MODALIDAD DE CONCLUSIÓN & Total \\
\hline LAUDO CONDENATORIO & 40 \\
\hline LAUDO ABSOLUTORIO & 67 \\
\hline FALTA DE INTERÉS PROCESAL & 668 \\
\hline CONVENIO FIRMADO & 2082 \\
\hline $\begin{array}{l}* \text { OTRA MODALIDAD DE } \\
\text { CONCLUSIÓN }\end{array}$ & 1814 \\
\hline TOTAL Fuente: Elaboración Propia. & 10 \\
\hline \multicolumn{2}{|c|}{} \\
\hline
\end{tabular}

De la información de la Tabla 2 se desprende que el 44,60\% de las quejas atendidas con relación a la especialidad gineco-obstetra llegaron a la firma de un convenio. ${ }^{11}$ Solo el $0,86 \%$ de las quejas atendidas por dicha comisión concluyeron mediante un laudo condenatorio, mientras que el $1,44 \%$ de las quejas atendidas se resolvió mediante laudo absolutorio. ${ }^{12}$ Un $14,30 \%$ se desestimaron por falta de interés procesal. ${ }^{13} \mathrm{Y}$, por último, un $38,80 \%$ de las quejas atendidas sencillamente concluyeron por improcedencia de la queja, no conciliación sin llegar a laudo, fallecimiento de la quejosa, entre otros.

Otro dato a considerar son las recomendaciones emitidas por la CNDH en México (CNDH), misma entidad que en el año 2018 emitió un total de ocho recomendaciones respecto a hechos que a su consideración son constitutivos de violencia obstétrica. Si bien ninguna de ellas versa sobre hechos acontecidos en el Estado de Veracruz, la existencia de estas nos debe permitir percatarnos que la violencia obstétrica se sigue manifestando en el país. Más allá de esto, es imprescindible destacar que en las ocho recomendaciones realizadas en el año 2018 por la CNDH ninguna de ellas versa solo sobre la violencia

\footnotetext{
${ }^{10}$ La información utilizada para realizar la tabla fue obtenida del oficio CONAMED-DGCI-55-2018, mediante el cual la Comisión Nacional de Arbitraje Médico da respuesta a una solicitud directa de información a través de la Plataforma Nacional de Transparencia de México. Se realizó la solicitud de información debido a que en la publicación de los Cuadros Estadísticos de Controversias de Salud 19962017, la información no es proporcionada.

${ }^{11}$ Es importante señalar que la CONAMED no informa si el convenio ha sido cumplimentado, si se les da alguna clase de seguimiento o cuales son los términos de los convenios firmados.

${ }^{12}$ Por laudo condenatorio se entiende como una resolución del procedimiento de queja por parte de la comisión mediante la cual condena y sanciona las acciones desplegadas por el personal de salud implicado. En tanto que un laudo absolutorio se entiende como la resolución posterior al procedimiento de queja, en la que la comisión considero que el personal médico implicado no tuvo responsabilidad en los hechos motivos de la queja.

${ }^{13}$ En este caso, debemos entender como falta de interés procesal, el hecho de que la parte afectada no dio seguimiento a la queja presentada a la comisión, por lo cual esta última desecha la queja.
} 
obstétrica que no produce la muerte del producto o secuelas posteriores al mismo como se puede observar en la Tabla 3, es decir, parece que solo es en el caso de que se presenten cualquiera de estas situaciones la CNDH emite recomendación sobre el asunto, pese a que se reconoce que dentro de sus recomendaciones visibiliza la violencia obstétrica física, verbal e institucional. Sería interesante que la CNDH emitiera recomendación sobre algún caso de violencia obstétrica en el cual ni el producto o la madre pierdan la vida, o que tengan secuelas por la mala praxis médica durante el parto, sino más bien que visibilizara que, aun cuando el parto fuere exitoso, los tratos proporcionados a la mujer dentro de su embarazo, parto y puerperio inmediato podrían considerarse como violencia obstétrica, que, en vista de los hechos de los que realizó las recomendaciones señaladas, se podría aludir que en muchas ocasiones son prácticas sistemáticas. ${ }^{14}$

Tabla 3. Recomendaciones por parte de la comisión nacional de los derechos humanos de México en las cuales se habla sobre casos de violencia obstétrica.

\begin{tabular}{|l|l|l|}
\hline $\begin{array}{l}\text { NÚMERO DE } \\
\text { RECOMENDACIÓN }\end{array}$ & ESTADO & $\begin{array}{l}\text { ACONTECIMIENTO PRINCIPAL } \\
\text { QUE MOTIVA LA } \\
\text { RECOMENDACIÓN }\end{array}$ \\
\hline $\mathbf{3 6 / 2 0 1 8}$ & $\begin{array}{l}\text { Ciudad de } \\
\text { México }\end{array}$ & $\begin{array}{l}\text { Secuelas neurológicas en el producto } \\
\text { por un mal parto. }\end{array}$ \\
\hline $\mathbf{4 0 / 2 0 1 8}$ & Quintana Roo & Muerte del producto. \\
\hline $\mathbf{5 2 / 2 0 1 8}$ & Chiapas & Muerte del producto. \\
\hline $\mathbf{5 4 / 2 0 1 8}$ & $\begin{array}{l}\text { Ciudad de } \\
\text { México }\end{array}$ & Muerte de uno de los productos. \\
\hline $\mathbf{5 5 / 2 0 1 8}$ & Tamaulipas & Muerte del producto. \\
\hline $\mathbf{5 6 / 2 0 1 8}$ & Michoacán & $\begin{array}{l}\text { Complicaciones médicas en el } \\
\text { producto debido a la inadecuada } \\
\text { atención en el parto. }\end{array}$ \\
\hline $\mathbf{5 8 / 2 0 1 8}$ & $\begin{array}{l}\text { Secuelas neurológicas en el producto } \\
\text { por un mal parto. }\end{array}$ \\
\hline $\mathbf{6 5 / 2 0 1 8}$ & México & $\begin{array}{l}\text { Muerte del producto. } \\
\text { Ciudad de }\end{array}$ \\
\hline
\end{tabular}

Fuente: Elaboración propia. ${ }^{15}$

De la tabla podemos observar que cuatro de ellas son emitidas contra actos de violencia obstétrica acontecidos en hospitales públicos de la Ciudad de México, y las

14 Por ejemplo, en varias de las recomendaciones la CNDH manifiesta que el personal médico sencillamente no prestaba atención a lo que comunicaba la víctima o simplemente no la atendían a tiempo. Otro ejemplo es que no le brindaban la información oportuna o existía indiferencia y malos tratos por parte del personal de salud.

${ }^{15}$ La información utilizada en la presente tabla se desprende del análisis de las recomendaciones realizadas por la Comisión Nacional de los Derechos Humanos en México en el año 2018, las cuales versan sobre hechos constitutivos de violencia obstétrica. 
otras cuatro por sucesos en el Estado de Chiapas, Quintana Roo y Tamaulipas. En ellas, con respecto a la violencia obstétrica, solicitan a la Secretaría de salud del Estado respectivo que: se tomen las medidas para que se realice la reparación del daño a las víctimas; se presente queja y se dé seguimiento a la misma ante la contraloría interna de los Estados respecto al actuar de los servidores públicos involucrados; se registre a las victimas ante el Registro Nacional de Victimas para que tengan acceso al Fondo de Ayuda Asistencia y Reparación Integral de la Comisión Ejecutiva de Atención a Víctimas; y, por último, que se diseñe un curso integral de capacitación a todos los servidores públicos adscritos al hospital donde aconteció la violencia, sobre capacitación y formación en materia de derechos humanos con un énfasis en el derecho a la protección de la salud y al derecho a una vida libre de violencia obstétrica. Es importante en relación a estas recomendaciones que en ninguno de los casos la Comisión hace referencia alguna a que se haga una denuncia respecto a la violación de los derechos reproductivos de las mujeres (por lo que respecta a las acontecidas en la Ciudad de México, en cuyo código penal se encuentra previsto el ilícito) o por el delito de violencia obstétrica en el caso de los hechos acontecidos en el Estado de Quintana Roo y Chiapas.

Un detalle curioso es el que independientemente de que en su recomendación $31 / 2017$, la Comisión ya considerare que la violencia física se manifestaba no solo en el abuso físico y abuso verbal, la recomendación 52/2018, se limite a indicar en su párrafo 71, que la violencia obstétrica tiene solo dos modalidades «física y psicológica» (Recomendación de la CNDH, 52/2018, de 31 de octubre de 2018).

\section{CONSIDERACIONES FINALES}

Como ya se he manifestado en otras oportunidades, la tipificación de la violencia obstétrica como único medio para la prevención y sanción de este tipo de violencia no es suficiente ni adecuada, ya que puede generar reticencias por parte de los profesionales de 
salud, de quienes se requiere más bien una participación para poder prevenir esta clase de violencia, cuestión respecto a la cual la criminalización puede lograr el efecto contrario. ${ }^{16}$

Además de la reticencia del personal de salud, debemos considerar que la violencia obstétrica también se puede presentar no por responsabilidad del profesional de salud, sino por las instituciones médicas, ya sea por exigir a su personal la aplicación de algún método en específico (como en los hospitales en que se prioriza la cesárea en lugar del parto natural) o que no cuenten con la cantidad de personal necesaria para poder brindar una atención adecuada. Por ello, nos encontramos que el delito de violencia obstétrica en México, por lo que respecta a los estados que la sancionan, no contempla sanción alguna para la violencia obstétrica institucional, dado que el tipo penal se limita a sancionar al profesional de salud. En el caso que comento, no debería ser penalmente responsable si la institución no le brinda las herramientas adecuadas.

El número de denuncias por violencia obstétrica, así como el número de recomendaciones por parte de la $\mathrm{CNDH}$, nos permite observar que la sociedad aún no ha internalizado el concepto de violencia obstétrica, o en su caso, la violencia obstétrica se encuentra normalizada. Para ello, más que establecer un tipo penal se podría hacer uso de los medios de comunicación masiva mediante documentales, telenovelas, noticieros o series televisivas en México, con la finalidad de cambiar la percepción del cómo debe ser la atención por parte de los profesionales de salud durante el embarazo, el alumbramiento y el puerperio, en la búsqueda de generar el rechazo social a toda practica vejatoria, pretendiendo a su vez informar a la sociedad que en caso de ser víctima de violencia obstétrica, se cuentan con los medios para exigir que se respeten sus derechos. Las campañas de sensibilización a las autoridades y a los profesionales de salud respecto a la violencia obstétrica deberían de extenderse a la sociedad en general, a efecto de que quien tenga conocimiento de un caso de este tipo de violencia pueda informarlo a las autoridades respectivas.

El hecho de establecer delitos que no se investiguen o que, al cometerse los ilícitos, no se llegue a un fallo condenatorio, demeritan más la confianza en el sistema judicial y

\footnotetext{
${ }^{16}$ Podemos recordar las marchas celebradas en México en el mes de abril del presente año, en el cual realizaron paro de labores exigiendo que no se criminalizara su profesión con relación a la detención de un médico en el Estado de Oaxaca por su presunta participación en el homicidio doloso de un menor de edad, fallecimiento que, a decir de la fiscalía de ese Estado, se debió a la mala praxis médica. Para más información véase: Hernández, Melissa. Protestan médicos por colegas encarcelados; marcharon en calles de Xalapa. En: El diario de Xalapa, 9 de abril de 2018.
} 
hace que permee en la sociedad la sensación de impunidad que, a su vez, hace que las personas que incurren en esas conductas se sientan amparados por la norma o refuercen la idea de que su actuar es el adecuado, y que las víctimas de violencia decidan no denunciar, o inclusive normalizar la violencia y considerar que lo que les ha acontecido no es ilícito o es adecuado. Por ello, se puede inferir que el hecho de que se tipificare la violencia obstétrica en el país sencillamente ha atendido más a dar cumplimiento a obligaciones internacionales, visto que la propia $\mathrm{CNDH}$, en sus recomendaciones no indica que se deba perseguir el ilícito de violencia obstétrica o violencia a los derechos reproductivos de las mujeres, en los casos en que se ha enterado de hechos que en los Estados que se han cometido son precisamente delitos.

Establecer la necesidad de prevenir la violencia obstétrica en los planes de desarrollo, o prever programas y manuales que procuren que el seguimiento al embarazo y el parto sea más humanizado, sin contar con mecanismos de seguimiento adecuado a su implementación, así como sanciones adecuadas a las instituciones médicas que no los observen, son sencillamente buenas intenciones o un discurso progresista del Estado, de los cuales no se obtendrá ningún resultado. 


\section{BIBLIOGRAFÍA}

Comisión Nacional de ARbitraje Médico (CONAMED). Cuadros Estadísticos de Controversias en Salud 1996-2017. Ciudad de México: CONAMED, 2018.

Comisión Nacional de ARbitraje MÉdico (CONAMED). Oficio CONAMED-DGCI55-2018. 27 de abril de 2018. Ciudad de México: CONAMED, 2018.

CORRAL, Guillermo. ¿Es necesario tipificar la violencia obstétrica? En: GoBERNATRICAS, Josefina; Boladeras, Margarita (eds.), El concepto «Violencia Obstétrica» y el debate actual sobre la atención al nacimiento. Barcelona: Tecnos, 2018, pp. 93104.

CRUZ, Sebastián, et al. El discurso de la violencia obstétrica en Chile. Taller de Metodologías Cualitativas Innovadoras en Investigación Psicosocial. Santiago de Chile: Escuela de Psicología U. de Chile, 2015.

CRUZ, Ángeles. Por cesárea, 47\% de nacimientos, el triple de lo recomendado por la OMS. En: La Jornada. 26 de octubre de 2015.

Distrito FEDERAL (MÉXICO). Decreto de 18 de marzo de 2011, por el que se reforma el código penal del Distrito Federal. En: GODF. 2011, décima séptima época, núm. 1056.

FisCALÍA GENERAL DEL ESTADO DE VERACRUZ. Dirección de Transparencia, Acceso a la Información y Protección de Datos Personales. Oficio 1649/2017. 31 de agosto de 2017. Veraet al, México.

HERNÁNDEZ, Melissa. Protestan médicos por colegas encarcelados; marcharon en calles de Xalapa. En: El diario de Xalapa. 9 de abril de 2018.

MARTIN, Julia; PAYÀ, Monserrat. Violencia Obstétrica: La lacra detrás del parto feliz. En: GoBERnA-TriCAS, Josefina; BoladerAs, Margarita (eds.), El concepto «Violencia Obstétrica» y el debate actual sobre la atención al nacimiento. Barcelona: Tecnos, 2018, pp. 69-73.

Mecanismo de Seguimiento de la Convención Interamericana para PReVenir, SANCIONAR y ERRADicAR la Violencia CONTRA LA Mujer (MESECVI). Segundo Informe Hemisférico sobre la Implementación de la Convención Interamericana para Prevenir, Sancionar y Erradicar la Violencia contra la Mujer. Washington: MESECVI, 2012. 
Poder Judicial del Estado de Veracruz. Unidad de Transparencia y Acceso a la Información Pública. Oficio UTAIPPJE/406/2017. 18 de agosto de 2017. Veracruz, México.

Recomendación de la Comisión Nacional de los DeRechos Humanos (MéXico) 06/2017, de 28 de febrero de 2017.

Recomendación de la Comisión Nacional de los Derechos Humanos (México) 31/2017, de 31 de julio de 2017.

Recomendación de la Comisión Nacional de los Derechos Humanos (MéXico) 36/2018, de 17 de octubre de 2018.

Recomendación de la Comisión Nacional de los Derechos Humanos (MÉXico) 40/2018, de 24 de octubre de 2018.

Recomendación de la Comisión Nacional de los Derechos Humanos (MéXico) 52/2018, de 31 de octubre de 2018.

ReCOMENDACión de la Comisión Nacional de los DeRechos Humanos (MÉXico) 54/2018, de 31 de octubre de 2018.

ReCOMENDACión de la Comisión Nacional de los DeRechos Humanos (MÉXICO) 55/2018, de 9 de noviembre de 2018.

Recomendación de la Comisión Nacional de los Derechos Humanos (México) 56/2018, de 12 de noviembre de 2018.

Recomendación de la Comisión Nacional de los DeRechos Humanos (MÉXiCO) 58/2018, de 14 de noviembre de 2018.

Recomendación de la Comisión Nacional de los Derechos Humanos (MÉxico) 65/2018, de 30 de noviembre de 2018.

VERACRUZ (MÉXICO). Decreto número 824, de 2 de abril de 2010, por el que se reforma el código penal. (GOEV, tomo CLXXXI, núm.107). 